OPEN ACCESS

Edited by:

Johnny Padulo,

Università degli Studi eCampus, Italy

Reviewed by:

Lauri Stenroth

University of Eastern Finland, Finland

Olivier Seynnes,

Norwegian School of Sport Sciences,

Norway

${ }^{*}$ Correspondence:

Alexandre Foure

alexandre.foure@hotmail.fr

Specialty section

This article was submitted to

Exercise Physiology,

a section of the journal

Frontiers in Physiology

Received: 20 June 2016

Accepted: 14 July 2016

Published: 27 July 2016

Citation:

Fouré A (2016) New Imaging Methods for Non-invasive Assessment of

Mechanical, Structural, and Biochemical Properties of Human Achilles Tendon: A Mini Review.

Front. Physiol. 7:324.

doi: 10.3389/fphys.2016.00324

\section{New Imaging Methods for Non-invasive Assessment of Mechanical, Structural, and Biochemical Properties of Human Achilles Tendon: A Mini Review}

\author{
Alexandre Fouré * \\ Aix-Marseille Université, Centre National de la Recherche Scientifique, Centre de Résonance Magnétique Biologique et \\ Médicale CRMBM UMR 7339, Marseille, France
}

The mechanical properties of tendon play a fundamental role to passively transmit forces from muscle to bone, withstand sudden stretches, and act as a mechanical buffer allowing the muscle to work more efficiently. The use of non-invasive imaging methods for the assessment of human tendon's mechanical, structural, and biochemical properties in vivo is relatively young in sports medicine, clinical practice, and basic science. Non-invasive assessment of the tendon properties may enhance the diagnosis of tendon injury and the characterization of recovery treatments. While ultrasonographic imaging is the most popular tool to assess the tendon's structural and indirectly, mechanical properties, ultrasonographic elastography, and ultra-high field magnetic resonance imaging (UHF MRI) have recently emerged as potentially powerful techniques to explore tendon tissues. This paper highlights some methodological cautions associated with conventional ultrasonography and perspectives for in vivo human Achilles tendon assessment using ultrasonographic elastography and UHF MRI.

Keywords: ultrasonography, elastography, magnetic resonance imaging, tendon stiffness, tendinopathy

\section{INTRODUCTION}

The mechanical properties of tendon are highly involved in muscle tension transmission to the skeleton and in the storage-recoil process of elastic potential energy (Alexander and Bennet-Clark, 1977; Roberts et al., 1997) playing an important role in daily activities and sport practices. Tendons exhibit non-linear viscoelastic behavior (Fung, 1981) which has a direct effect on the efficiency of muscular tension transmission to the skeleton and limits stress on muscle (e.g., buffer effect of tendon to slow down eccentric velocity of muscle contraction in jump landing). Tendon viscoelastic properties are highly influenced by the composition of tendinous tissues (Langberg et al., 2001; Kjaer et al., 2009; Thorpe et al., 2012) and especially collagens, proteoglycans, and water (Kjaer, 2004; Wang, 2006; Connizzo et al., 2013). While it is very difficult to dissociate both elastic and viscous behaviors, it has been shown that the crosslinking of collagens increases the elastic modulus and reduced strain at failure (Thompson and Czernuszka, 1995) whereas several components of the extracellular matrix (Kjaer, 2004), especially water (van der Rijt et al., 2006) and proteoglycans (Yoon and Halper, 2005), can be associated with the viscous behavior observed during assessment in vitro (Silver et al., 2002; Gautieri et al., 2012). 
Over the last two decades, ultrasonography has remained the gold standard method to assess tendon structural and mechanical properties non-invasively in vivo (Fukashiro et al., 1995; Maganaris and Paul, 2000, 2002; Maganaris, 2002; Magnusson, 2002; Kubo et al., 2002c; Arampatzis et al., 2005). Effects of aging (Magnusson et al., 2003a; Mademli and Arampatzis, 2008; Kubo et al., 2014), gender (Kubo et al., 2003; Magnusson et al., 2007; Westh et al., 2008), rehabilitation (Arya and Kulig, 2010; Geremia et al., 2015), bedrest (Kubo et al., 2000, 2004) or chronic interventions such as training (Kubo et al., 2002a,b; Fouré et al., 2010, 2013) have been abundantly studied. From an experimental point of view, structural and mechanical tendon properties are commonly assessed in vivo from the forceelongation and stress-strain relationships (Figure 1) obtained with a constant increase in tension (i.e., loading phase) applied on the tendon due to a passive stretching of muscle tendon unit (Morse et al., 2008) or a controlled isometric contraction (Fouré et al., 2010). Tendon length change is then measured from ultrasound images and synchronized to external torque measured in most cases by a dynamometer (Maganaris and Paul, 2002). In addition, tendon hysteresis can also be determined from the relationship including loading and unloading phases (Maganaris and Paul, 2000). Many methodological strategies associated with the experimental conditions have been reported, discussed and reviewed (Maganaris, 2005; Arampatzis et al., 2008; Finni et al., 2013; Lichtwark et al., 2013; Seynnes et al., 2015). The real time evaluation of tendon length changes used in the non-invasive assessment of tendon structural properties during contraction is a considerable advantage of ultrasound imaging. However, there remain associated methodological issues from the limited spatial coverage of ultrasound probes (e.g., 2D assessment of 3D stain, restricted planar field of view) and the data normalization required to calculate force or stress values from external torque measurements and strain on the basis of tendon elongation. Indeed, most of the published studies chose arbitrary values for tendon cross sectional area (CSA) and slack length (Maganaris, 2002; Magnusson et al., 2003b; Arya and Kulig, 2010) when it is now well known that the Achilles tendon slack length does not correspond to the tendon length when the ankle joint angle is at $90^{\circ}$ (Nordez et al., 2010; Hug et al., 2013) and that CSA is not homogeneous along the tendon (Bohm et al., 2015; Lenskjold et al., 2015). Other limitations were previously reviewed in detail (Seynnes et al., 2015). Thus, the mechanical properties of tendinous tissues assessed from stressstrain relationship estimated from external torque measurement and ultrasound imaging can appear inaccurate considering uncontrolled or arbitrary fixed parameters and variability among individuals. To avoid these methodological issues associated with the experimental design and choices in the normalization of the parameters, additional special imaging technologies have been developed to quantitatively assess biomechanical and biochemical properties of tissues.

The goal of this paper is to highlight the emergence of new imaging methodologies as powerful tools for the non-invasive exploration of tendinous tissues. Although for now elastography is mainly used on skeletal muscle (Bensamoun et al., 2006; Hug et al., 2015), technical evolution of ultrasonographic devices

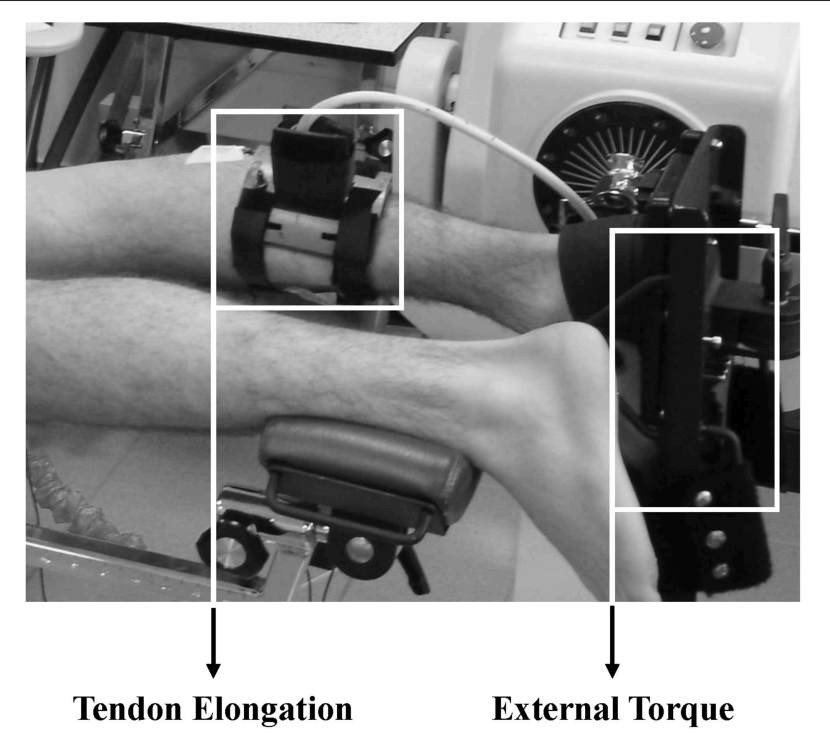

Relative contribution of plantar flexor muscles

Tendon Force-Length relationship

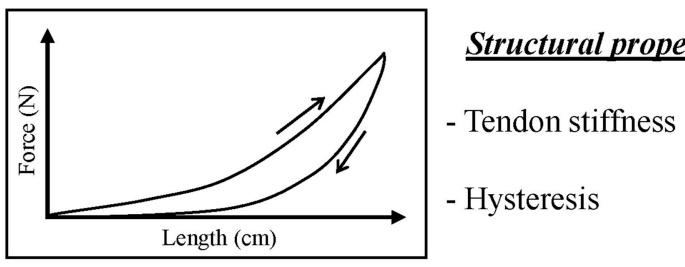

\section{Additional Estimations Measurements \\ Tendon cross sectional area}

Tendon Stress-Strain relationship

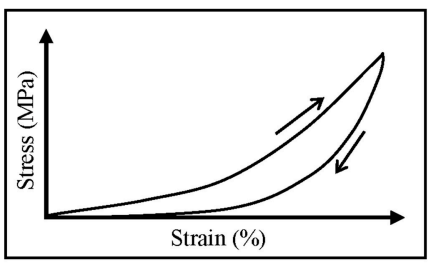

Mechanical properties

- Tendon elastic modulus

- Hysteresis

FIGURE 1 | Experimental position of the subject during assessment of Achilles tendon structural and mechanical properties with ultrasonography and dynamometry. During passive motion of ankle joint or isometric ramp of plantar flexors contraction, displacement of myotendinous junction is recorded with ultrasonography while external torque is measured with an isokinetic dynamometer. Considering additional measurements of tendon length or estimations of lever arm and the relative contribution of muscles involved in tendon elongation, force-length relationship is established in order to characterize tendon stiffness (structural property, dependent of tendon geometry) and hysteresis in case of loading-unloading cycle. In addition, stress-stain relationship of the tendon can be determined from initial tendon length and tendon cross sectional area. Elastic modulus of tendinous tissues can further be assessed (mechanical property intrinsically related to the tissue, independent of the geometry). 
increases applications of elastography on tendon (HelfensteinDidier et al., 2016). This mini-review is focused on recent developments and applications of ultrasound elastography and ultra-high field magnetic resonance imaging for the non-invasive assessment of tendon biomechanical and biochemical properties (Figure 2).

\section{ASSESSMENT OF ACHILLES TENDON BIOMECHANICAL PROPERTIES WITH ELASTOGRAPHY}

Over the last decade, there has been growing evidence that elastography may be a useful tool in detecting subtle changes in musculotendinous mechanical properties that occur early in the course of an injury or disorder.

\section{Ultrasound Elastography}

Application of ultrasound elastography on skeletal muscle has been widely developed (Bercoff et al., 2004; Gennisson et al., 2013; Brandenburg et al., 2014). As reported in a recent review, "supersonic shear wave imaging (SSI) is the current state of the art in ultrasound elastography” (Hug et al., 2015). This latter technology consists in applying a stress on soft tissue through the acoustic radiation force of a long burst of focused ultrasound pulses (i.e., ultrasound push beams) producing a shear wave observed via high frame pulse-echo ultrasound imaging. The time shift of the ultrasound echo is then used to measure deformation associated with the displacement of the shear wave in the tissue. Shear wave elastography is a quantitative method to measure the shear wave velocity to estimate the localized elastic properties of tissue in vivo (Bercoff et al., 2004; Brandenburg et al., 2014). Considering tissue as elastic and homogeneous, the shear modulus $(\mu, \mathrm{kPa})$ is calculated using the equation: $\mu=$ $\rho V^{2}$, where $\rho$ is the density of the tissue $\left(\mathrm{kg} \cdot \mathrm{m}^{-3}\right)$ and $V$ the shear wave velocity $\left({\mathrm{m} . \mathrm{s}^{-1}}^{\text {) }}\right.$ (Bercoff et al., 2004; HelfensteinDidier et al., 2016). Considering isotropic locally homogeneous and quasi-incompressible biological tissues, Young's modulus $(E)$ can be estimated from the shear modulus using the following equation: $E \approx 3 \mu$ (Bercoff et al., 2004; Hug et al., 2015). While many studies have recently assessed muscle mechanical properties with SSI (Lacourpaille et al., 2014; Hug et al., 2015; Le Sant et al., 2015), this method was also used to determine the slack length of Achilles tendon during passive stretches of the ankle (Hug et al., 2013). Although measurements saturate at relatively low tension levels in the latter study $\left(\sim 20^{\circ}\right.$ in plantar flexion during passive stretching) due to the high shear wave speed

A

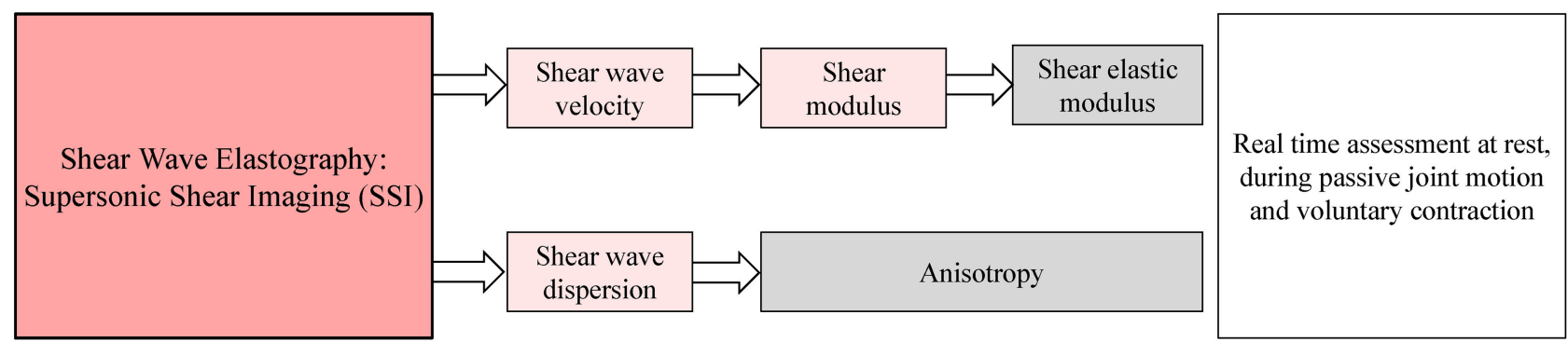

B

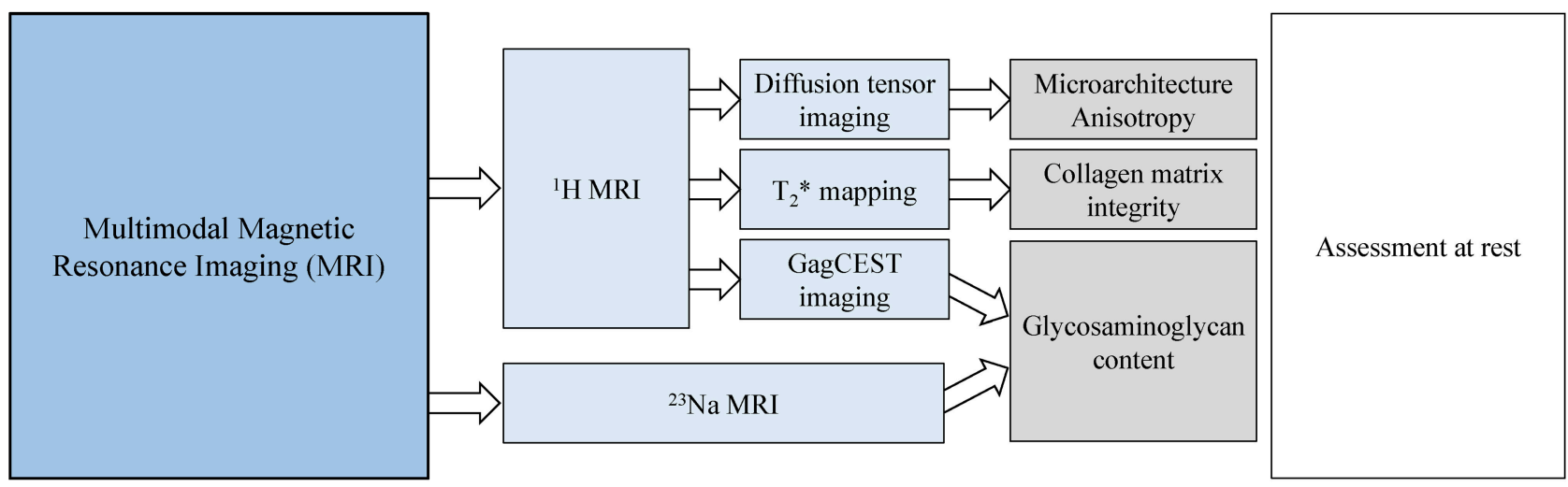

FIGURE 2 | Schematic representation of emergent imaging techniques for the assessment of tendon biomechanical properties and biochemical composition. (A) Supersonic Shear Imaging can be used to assess shear elastic modulus and anisotropy of the tendon via the analysis of shear wave velocity and dispersion. (B) Multimodal Magnetic Resonance Imaging can be used to quantify glycosaminoglycan content, assess collagen matrix integrity and characterize diffusivities of water molecules inside tendon at rest. 
observed in stiff tissue such as tendon, feasibility, and accuracy of the slack length measurement with SSI was demonstrated (Hug et al., 2013). Given that muscle and tendon slack lengths correspond to different ankle angles, the latter study also shed light on the complex interaction between muscles and Achilles tendon during passive motion of lower limbs.

In the last 5 years, a growing interest in tendon mechanical properties assessment with SSI can be observed. For instance, differences in shear wave velocities have been highlighted among musculotendinous structures (i.e., tendon, aponeurosis, muscle) and according to age (Slane et al., 2015, 2016; Turan et al., 2015). Arda et al. (2011) showed that Young's modulus at rest is higher in Achilles tendon $(52 \pm 25 \mathrm{kPa}$ in transverse plane and $74 \pm$ $46 \mathrm{kPa}$ in longitudinal plane) as compared to the gastrocnemius muscle (11 $\pm 4 \mathrm{kPa}$ in longitudinal plane). It is noteworthy that a high inter-individual variability was shown (Arda et al., 2011) and shear wave elastography measurements were dependent on imaging plane (Chino et al., 2015). From a methodological point of view, shear wave elastography in soft tissues such as muscle and tendon must be performed with the lightest transducer pressure (Kot et al., 2012). In addition, both transducer positioning and limb posture have to be carefully checked to limit spatial variations in Achilles tendon shear wave speed (DeWall et al., 2014). Aubry et al. (2013) found an effect of ankle joint angle on shear wave propagation velocity. In the latter study, Achilles tendon anisotropy was calculated on the basis of the difference in the shear wave speed determined in the axial and the longitudinal planes (Aubry et al., 2013). The relative anisotropy coefficient and elastic modulus of Achilles tendon were increased when the ankle was dorsiflexed (Aubry et al., 2013). Brum et al. (2014) assessed the elastic anisotropy of the human Achilles tendon using shear wave dispersion analysis. In this study, shear wave velocity dispersion was measured in both axial and longitudinal planes to the Achilles tendon fiber orientation. Given that the shear wavelength in the longitudinal direction is five times larger than the mean tendon thickness, the wave propagation is guided along the tendon by successive reflections at the tendon boundaries (Brum et al., 2014). Hence, the use of a specific model was needed to characterize tendon viscoelastic properties taking into account the latter phenomenon. On the basis of the shear wave dispersion analysis developed by Brum et al. (2014), HelfensteinDidier et al. (2016) found that Achilles tendon shear modulus increases with passive dorsiflexion. Regardless of ankle angle, the shear modulus was significantly higher in the proximal region of the Achilles tendon as compared to the more distal one. Very good reproducibility results were reported with coefficients of variation lower than $1 \%$ and shear modulus values determined with the shear wave dispersion analysis and conventional shear wave elastography technique were highly correlated $(r=0.844, P$ $<0.001$ ). This indicates that SSI can be used to compare tendon mechanical properties determined from shear modulus and then shear elastic modulus across populations and could have a clinical relevance in tendinopathy (Helfenstein-Didier et al., 2016). It is noteworthy that the assumption associated to the SSI technique of an elastic and homogeneous medium is not necessarily entirely correct in the tendon. In fact, the anisotropic characteristics and inter-individual variabilities in microarchitecture of the tendon could limit interpretations when comparing healthy individuals or assessing the effects of interventions with SSI. In addition, measurement of shear elastic modulus with SSI is based on a constant tendon density which can be different in healthy and pathologic tendons. Indeed, it was clearly shown that tendinopathies can induce changes in the structural organization and biochemical composition of tendinous tissues (de Mos et al., 2007; Pingel et al., 2014) leading to a potential inaccuracy in shear elastic modulus assessment. Nevertheless, relevant clinical assessment of tendon disorders assessed with SSI have already been reported in a recent review (Klauser et al., 2014). For instance, lower tendon stiffness was found in patient with torn Achilles tendon as compared to healthy subjects (Chen et al., 2013) suggesting that shear wave elastography provides relevant biomechanical information for Achilles tendon function assessment. In addition, a lower shear wave velocity was found in stretched Achilles tendon of patients with tendinopathy as compared to healthy control subjects whereas no significant difference was reported in Achilles tendon anisotropy (Aubry et al., 2015).

Furthermore, 3D ultrafast ultrasound imaging for the 3D mapping of stiffness, tissue motion, and flow in humans was recently demonstrated in vivo as a future new clinical application of ultrasound with reduced intra- and inter-observer variability (Provost et al., 2014). On that basis, it can be expected that methodological developments for 3D assessment of mechanical properties in soft tissues with SSI will continue to progress. However, for now, the 3D exploration of musculoskeletal system with shear wave elastography remains associated with nuclear magnetic resonance techniques (Muthupillai et al., 1995).

\section{Magnetic Resonance Elastography}

In comparison to ultrasound elastography, magnetic resonance elastography (MRE) has the advantages of full 3D acquisition and can explore deep muscles with large spatial coverage in a well-defined and reproducible coordinate system (Gennisson et al., 2010). MRE typically uses vibrations of a single frequency within the audio frequency range. The shear waves are generated by an electro-mechanical transducer on the surface of the skin and the tissue motion is measured using MRI technique called phase-contrast MRI (Muthupillai et al., 1995). MRE allows assessment of mechanical properties of soft tissues (Mariappan et al., 2010) such as skeletal muscle (Dresner et al., 2001; Bensamoun et al., 2006). MRE has been used to investigate noninvasively lower limb skeletal muscles mechanical properties and detect abnormalities in patients with neuromuscular disorders as compared to matched control subjects (Basford et al., 2002). However, in early studies on skeletal muscle, neither viscosity nor anisotropy were taken into account to assess mechanical properties. Subsequent MRE studies demonstrated the high anisotropy of the muscle tissue (Papazoglou et al., 2005). Recently, multi-frequency MRE and rheological models were used to assess the viscoelastic shear properties of thigh muscles in passive condition in vivo (Chakouch et al., 2016). So far, MRE has only been used on skeletal muscle and no exploration on tendon has been performed in vivo. Indeed, very stiff tissues such as tendon (in comparison to skeletal muscle) require much higher 
vibration frequencies for mechanical properties assessment with MRE. Current MRI scanners do not have gradient hardware that is capable of encoding wave motion at such high frequencies (Mariappan et al., 2010). These limitations may be addressed in the future with specialized hardware solutions and development of ultra-high field (UHF) MRI clinical scanners with special high-speed imaging techniques (Glaser et al., 2006).

Considering that the intrinsic mechanical properties of the tendinous tissues are closely related to the tendon composition, UHF MRI appears a powerful imaging technique to assess microstructural and biochemical parameters closely linked to the viscoelastic behavior of the Achilles tendon; meanwhile, technological development is still necessary for the direct assessment of the tendon mechanical properties with MRE.

\section{ASSESSMENT OF ACHILLES TENDON BIOCHEMICAL AND STRUCTURAL PROPERTIES WITH MRI}

Magnetic resonance imaging allows an accurate assessment of biochemical composition and microstructure of musculoskeletal tissues. However, visualization of the tendon remains difficult due to a very short transverse relaxation time (i.e., $\mathrm{T}_{2}<1.5 \mathrm{~ms}$ ) leading to a partial or total disappearance of signal in the tendon with relatively long echo time used in conventional clinical MRI sequences (Gatehouse and Bydder, 2003). Thus, special MR sequences are required to acquire signal from the tendon. The most frequently used sequence in the recent studies consisted in a quantitative imaging of tendon using ultrashort echo time (UTE; Robson et al., 2004; Juras et al., 2012b) and variable echo time sequences (Song and Wehrli, 1998; Juras et al., 2013b).

Furthermore, improved signal to noise ratio (SNR) in emerging 7 Tesla (7T) whole body MRI scanners-SNR being proportional to the field strength-provides opportunities for easier examinations of musculoskeletal structures (Trattnig et al., 2015) and especially Achilles tendon (Trattnig et al., 2012). Hence, many studies are now using 7T-MRI to explore tendon structure and quantitatively assess structural and biochemical properties in vivo (Trattnig et al., 2012). For instance, Han et al. succeeded in using high-resolution 3D UTE sequence to visualize the Achilles tendon microstructure in human healthy volunteers (Han et al., 2014).

\section{Tendon Relaxation Constants}

Quantitative MRI is widely used to characterize potential alteration of musculoskeletal tissues. Change in $\mathrm{T}_{2}{ }^{*}$ relaxation time is commonly assessed in skeletal muscle to quantify the effects of edema/inflammation processes associated with acute injury (Fouré et al., 2015b) or muscle disease (Arpan et al., 2013).

Despite the very short transverse relaxation time of the tendon, $\mathrm{T}_{2}{ }^{*}$ is the most popular relaxation constant used to detect potential collagen matrix alteration. Using variable echo time sequence, the bi-exponential $\mathrm{T}_{2}{ }^{*}$ signal decay has been analyzed in healthy subjects and patients with tendinopathies (Juras et al., 2012b, 2013b). A strong correlation between clinical score and the short component $\mathrm{T}_{2}{ }^{*}$ of pathologic tendon was demonstrated at $3 \mathrm{~T}$ (Juras et al., 2013b). It is noteworthy that the increased SNR at $7 \mathrm{~T}$ can provide higher accuracy of $\mathrm{T}_{2}{ }^{*}$ calculation as compared to $3 \mathrm{~T}$ (Juras et al., 2012b). Although the short component of $\mathrm{T}_{2}{ }^{*}$ was shown to be a robust and promising biomarker of tendon structural alterations (Chang et al., 2016), other parameters such as glycosaminoglycan content appear to be more specific to the changes in biochemical properties of injured or pathologic tendon.

The $\mathrm{T}_{2}$ assessment of supraspinatus tendon was shown to be reproducible (Anz et al., 2014) but it was only assessed on healthy volunteers and no data are available to check the sensitivity of this parameters to structural and/or biochemical changes in patients with tendinopathies and tendon injuries.

Another constant associated to both transverse and longitudinal relaxation times of the tissue $\left(\mathrm{T}_{1} \rho\right)$ is also used to assess musculoskeletal tissues (Wang and Regatte, 2015) and especially cartilage (Regatte et al., 2003). However, only one study has assessed Achilles tendon $\mathrm{T}_{1} \rho$ of cadaveric specimens and healthy control subjects using a $2 \mathrm{D}$ UTE $\mathrm{T}_{1} \rho$ sequence (Du et al., 2010). For now, $\mathrm{T}_{1} \rho$ is mostly used in basic sciences and does not yet have widespread clinical use.

\section{Glycosaminoglycans Content Assessment}

The detection of biochemical changes can help the early diagnosis of tendinopathy (Samiric et al., 2009). An increased amount of proteoglycan content in the extracellular matrix was reported in human pathologic tendons (Fu et al., 2007; Parkinson et al., 2010). The sulfate and carboxyl groups associated with glycosaminoglycans (GAG) provide proteoglycans with a net negative charge, attracting molecules with positive charge such as sodium ions. Thus, a strong correlation was reported between GAG content and sodium MRI at UHF in ex vivo tendon (Juras et al., 2013a). The increased GAG content in the tendon was also correlated with the Victorian Institute Sport Assessment (VISA) score (Attia et al., 2014), a widely used outcome measure of functional status and pain level. Expression of several proteoglycans typically associated with GAGs such as decorin, versican, and aggrecan were found to be higher in pathologic patellar tendon as compared to controls (Attia et al., 2014).

Sodium MRI is an imaging technique based on detection of ${ }^{23} \mathrm{Na}$ nuclei used to quantify sodium content in biological tissues which can provide an indirect quantification of tendon GAG content. A higher tendon sodium SNR was also reported in patients with tendinopathy as compared to healthy control (Juras et al., 2012a). Sodium MRI appears as a powerful and non-invasive method to detect early biochemical changes in tendinopathy. However, sodium imaging requires an MR system with multinuclear capabilities and dedicated sodium antenna coils. To bypass this issue, chemical exchange saturation transfer (CEST) can be used to assess GAG content (GagCEST) in the tendon. The method was first developed in cartilage (Schmitt et al., 2011) and provided an index of GAG content on the basis of chemical exchange between bulk water protons and protons bound to solutes (Guivel-Scharen et al., 1998). This method is emerging as a relevant alternative to sodium MRI but requires complex image post-processing. In addition, for accurate quantification of GagCEST effects, it is essential 
to account for inhomogeneities of the static magnetic field $\mathrm{B}_{0}$ and radiofrequency field $\mathrm{B}_{1}$. Nevertheless, reproducible measurements in knee cartilage of healthy volunteers have been recently provided (Schreiner et al., 2016).

So far, only one study has assessed Achilles tendon biochemical properties with multimodal MRI (i.e., $\mathrm{T}_{2}{ }^{*}$ mapping, sodium MRI and GagCEST; Juras et al., 2015). This study assessed effects of ciprofloxacin intake on Achilles tendon properties of seven healthy males. It was previously shown that fluoroquinolones such as ciprofloxacin can increase risk of tendon injuries (Stephenson et al., 2013). While no significant change in morphology and collagen matrix was detected with $\mathrm{T}_{2}{ }^{*}$ mapping, a decrease in GAG content was seen 10 days after ciprofloxacin intake using both sodium MRI and GagCEST. This biochemical change was not associated with clinical symptoms of tendon injury. Therefore, multimodal MRI could potentially be used to detect onset of abnormal change in tendon GAG content representing one of the early stages of tendinopathy.

It was previously shown that tendon stiffness assessed with ultrasonography and dynamometry is decreased in patients with tendinopathies (Arya and Kulig, 2010). In addition, changes in extracellular matrix highly influence tendon function (Kjaer, 2004). Given that stiffness is a structural characteristic of the tendon, dependent on the intrinsic mechanical properties of the tissues (i.e., related to the tendon biochemical composition) and the microarchitecture of the tendon, it appears relevant to concomitantly assess tendon biochemical and microarchitectural properties of the tendon.

\section{Microarchitecture Characterization}

Diffusion tensor imaging (DTI) is sensitive to changes in the microstructural architecture of biological tissues. Cell membranes and other solid structures restrict water diffusion leading to anisotropic diffusion. The integrity of tissues is then assessed by the predominant direction, intensity and isotropic characteristics of water diffusion within the biological structure. For instance, DTI is used in skeletal muscle to determine potential exercise-induced structural alterations (Fouré et al., 2015a) and potential changes in muscle architecture (i.e., fiber length and pennation angle) with muscle fiber tractography

\section{REFERENCES}

Alexander, R. M., and Bennet-Clark, H. C. (1977). Storage of elastic strain energy in muscle and other tissues. Nature 265, 114-117. doi: 10.1038/2 $65114 \mathrm{a} 0$

Anz, A. W., Lucas, E. P., Fitzcharles, E. K., Surowiec, R. K., Millett, P. J., and Ho, C. P. (2014). MRI T2 mapping of the asymptomatic supraspinatus tendon by age and imaging plane using clinically relevant subregions. Eur. J. Radiol. 83, 801-805. doi: 10.1016/j.ejrad.2014.02.002

Arampatzis, A., Monte, G. D., and Karamanidis, K. (2008). Effect of joint rotation correction when measuring elongation of the gastrocnemius medialis tendon and aponeurosis. J. Electromyogr. Kinesiol. 18, 503-508. doi: 10.1016/j.jelekin.2006.12.002

Arampatzis, A., Stafilidis, S., Demonte, G., Karamanidis, K., Morey-Klapsing, G., and Bruggemann, G. P. (2005). Strain and elongation of the human gastrocnemius tendon and aponeurosis during maximal plantarflexion effort. J. Biomech. 38, 833-841. doi: 10.1016/j.jbiomech.2004.04.031
(Cotten et al., 2015). For now, only a few studies have assessed anisotropy/microarchitecture of the tendon (Momot et al., 2010) in animals (Wellen et al., 2005; Helmer et al., 2006; Gupta et al., 2010) and in humans (Sarman et al., 2015). Since tendon has a short transverse relaxation time, methodological developments are needed to obtain shorter echo time than those available on conventional clinical scanners. New methodologies have recently been presented in the ISMRM annual meeting (He et al., 2016; Ma et al., 2016). Although methodological and experimental issues such as dependence of tendon fibers orientation in the static magnetic field (i.e., magic angle effect) have to be resolved, there is likely to be additional methodological developments for tendon microarchitecture assessment with UHF MRI and growing interest of the MR community for tendon assessment.

\section{CONCLUSION}

Non-invasive imaging methods for the assessment of human Achilles tendon in vivo have been widely adopted in the two last decades. Democratization in the use of ultrasonographic devices and technical developments in both ultrasound and MRI widen the perspectives for tendon assessment in the clinical context and for basic science. For now, ultrasonography is the most popular tool to assess tendon structural properties. However, the development of elastography based on ultrasound and MRI appears complementary in assessing tendon biomechanical and biochemical properties. While ultrasound elastography allows local assessment of the tendon in real-time and potentially during passive (i.e., passive joint motion) or active (i.e., muscle contraction) stretch, multimodal MRI can accurately assess tendon structural and biochemical properties in three dimensions at rest. Further studies associating quantitative MRI and elastography are needed in order to assess non-invasively the mechanical, structural and biochemical properties of the Achilles tendon and lead to clinical applications for diagnosis, prognosis and follow-up of tendinopathies and tendon injuries.

\section{AUTHOR CONTRIBUTIONS}

AF has designed, written, and approved this manuscript.
Arda, K., Ciledag, N., Aktas, E., Aribas, B. K., and Kose, K. (2011). Quantitative assessment of normal soft-tissue elasticity using shear-wave ultrasound elastography. AJR Am. J. Roentgenol. 197, 532-536. doi: 10.2214/AJR.10.5449

Arpan, I., Forbes, S. C., Lott, D. J., Senesac, C. R., Daniels, M. J., Triplett, W. T., et al. (2013). T(2) mapping provides multiple approaches for the characterization of muscle involvement in neuromuscular diseases: a cross-sectional study of lower leg muscles in 5-15-year-old boys with Duchenne muscular dystrophy. NMR Biomed. 26, 320-328. doi: 10.1002/nbm.2851

Arya, S., and Kulig, K. (2010). Tendinopathy alters mechanical and material properties of the Achilles tendon. J. Appl. Physiol. 108, 670-675. doi: 10.1152/japplphysiol.00259.2009

Attia, M., Scott, A., Carpentier, G., Lian, O., Van Kuppevelt, T., Gossard, C., et al. (2014). Greater glycosaminoglycan content in human patellar tendon biopsies is associated with more pain and a lower VISA score. Br. J. Sports Med. 48, 469-475. doi: 10.1136/bjsports-2013-092633

Aubry, S., Nueffer, J. P., Tanter, M., Becce, F., Vidal, C., and Michel, F. (2015). Viscoelasticity in Achilles tendonopathy: quantitative assessment 
by using real-time shear-wave elastography. Radiology 274, 821-829. doi: 10.1148/radiol.14140434

Aubry, S., Risson, J. R., Kastler, A., Barbier-Brion, B., Siliman, G., Runge, M., et al. (2013). Biomechanical properties of the calcaneal tendon in vivo assessed by transient shear wave elastography. Skeletal Radiol. 42, 1143-1150. doi: 10.1007/s00256-013-1649-9

Basford, J. R., Jenkyn, T. R., An, K. N., Ehman, R. L., Heers, G., and Kaufman, K. R. (2002). Evaluation of healthy and diseased muscle with magnetic resonance elastography. Arch. Phys. Med. Rehabil. 83, 1530-1536. doi: 10.1053/apmr.2002.35472

Bensamoun, S. F., Ringleb, S. I., Littrell, L., Chen, Q., Brennan, M., Ehman, R. L., et al. (2006). Determination of thigh muscle stiffness using magnetic resonance elastography. J. Magn. Reson. Imaging 23, 242-247. doi: 10.1002/jmri.20487

Bercoff, J., Tanter, M., and Fink, M. (2004). Supersonic shear imaging: a new technique for soft tissue elasticity mapping. IEEE Trans. Ultrason. Ferroelectr. Freq. Control 51, 396-409. doi: 10.1109/TUFFC.2004.1295425

Bohm, S., Mersmann, F., Marzilger, R., Schroll, A., and Arampatzis, A. (2015). Asymmetry of Achilles tendon mechanical and morphological properties between both legs. Scand. J. Med. Sci. Sports 25, e124-e132. doi: 10.1111/sms.12242

Brandenburg, J. E., Eby, S. F., Song, P., Zhao, H., Brault, J. S., Chen, S., et al. (2014). Ultrasound elastography: the new frontier in direct measurement of muscle stiffness. Arch. Phys. Med. Rehabil. 95, 2207-2219. doi: 10.1016/j.apmr.2014.07.007

Brum, J., Bernal, M., Gennisson, J. L., and Tanter, M. (2014). In vivo evaluation of the elastic anisotropy of the human Achilles tendon using shear wave dispersion analysis. Phys. Med. Biol. 59, 505-523. doi: 10.1088/0031-9155/59/ $3 / 505$

Chakouch, M. K., Pouletaut, P., Charleux, F., and Bensamoun, S. F. (2016). Viscoelastic shear properties of in vivo thigh muscles measured by MR elastography. J. Magn. Reson. Imaging 43, 1423-1433. doi: 10.1002/jmri.25105

Chang, E. Y., Healey, R. M., Biswas, R., Statum, S., Tran, B., Iwasaki, K., et al. (2016). "Correlation of mono-exponential and bi-exponential UTE-T2* analyses and biomechanics in human Achilles tendons," in Proceedings of the International Society for Magnetic Resonance in Medicine (ISMRM) (Singapore), 260.

Chen, X. M., Cui, L. G., He, P., Shen, W. W., Qian, Y. J., and Wang, J. R. (2013). Shear wave elastographic characterization of normal and torn achilles tendons: a pilot study. J. Ultrasound Med. 32, 449-455.

Chino, K., Kawakami, Y., and Takahashi, H. (2015). Tissue elasticity of in vivo skeletal muscles measured in the transverse and longitudinal planes using shear wave elastography. Clin. Physiol. Funct. Imaging. doi: 10.1111/cpf.12315. [Epub ahead of print].

Connizzo, B. K., Yannascoli, S. M., and Soslowsky, L. J. (2013). Structure-function relationships of postnatal tendon development: a parallel to healing. Matrix Biol. 32, 106-116. doi: 10.1016/j.matbio.2013.01.007

Cotten, A., Haddad, F., Hayek, G., Lefebvre, G., Dodre, E., and Budzik, J. F. (2015). Tractography: possible Applications in Musculoskeletal Radiology. Semin. Musculoskelet. Radiol. 19, 387-395. doi: 10.1055/s-0035-1563736

de Mos, M., Van El, B., Degroot, J., Jahr, H., Van Schie, H. T., Van Arkel, E. R., et al. (2007). Achilles tendinosis: changes in biochemical composition and collagen turnover rate. Am. J. Sports Med. 35, 1549-1556. doi: $10.1177 / 0363546507301885$

DeWall, R. J., Slane, L. C., Lee, K. S., and Thelen, D. G. (2014). Spatial variations in Achilles tendon shear wave speed. J. Biomech. 47, 2685-2692. doi: 10.1016/j.jbiomech.2014.05.008

Dresner, M. A., Rose, G. H., Rossman, P. J., Muthupillai, R., Manduca, A., and Ehman, R. L. (2001). Magnetic resonance elastography of skeletal muscle. J. Magn. Reson. Imaging 13, 269-276. doi: 10.1002/1522-2586(200102)13:2<269:: AID-JMRI1039>3.0.CO;2-1

Du, J., Carl, M., Diaz, E., Takahashi, A., Han, E., Szeverenyi, N. M., et al. (2010). Ultrashort TE T1rho (UTE T1rho) imaging of the Achilles tendon and meniscus. Magn. Reson. Med. 64, 834-842. doi: 10.1002/mrm.22474

Finni, T., Peltonen, J., Stenroth, L., and Cronin, N. J. (2013). Viewpoint: on the hysteresis in the human Achilles tendon. J. Appl. Physiol. 114, 515-517. doi: 10.1152/japplphysiol.01005.2012

Fouré, A., Duhamel, G., Wegrzyk, J., Boudinet, H., Mattei, J. P., Le Troter, A., et al. (2015a). Heterogeneity of muscle damage induced by electrostimulation: a multimodal MRI study. Med. Sci. Sports Exerc. 47, 166-175. doi: 10.1249/MSS.0000000000000397

Fouré, A., Le Troter, A., Guye, M., Mattei, J. P., Bendahan, D., and Gondin, J. (2015b). Localization and quantification of intramuscular damage using statistical parametric mapping and skeletal muscle parcellation. Sci. Rep. 5:18580. doi: $10.1038 /$ srep 18580

Fouré, A., Nordez, A., and Cornu, C. (2010). Plyometric training effects on Achilles tendon stiffness and dissipative properties. J. Appl. Physiol. 109, 849-854. doi: 10.1152/japplphysiol.01150.2009

Fouré, A., Nordez, A., and Cornu, C. (2013). Effects of eccentric training on mechanical properties of the plantar flexor muscle-tendon complex. J. Appl. Physiol. 114, 523-537. doi: 10.1152/japplphysiol.01313.2011

Fu, S. C., Chan, K. M., and Rolf, C. G. (2007). Increased deposition of sulfated glycosaminoglycans in human patellar tendinopathy. Clin. J. Sport Med. 17, 129-134. doi: 10.1097/JSM.0b013e318037998f

Fukashiro, S., Itoh, M., Ichinose, Y., Kawakami, Y., and Fukunaga, T. (1995). Ultrasonography gives directly but noninvasively elastic characteristic of human tendon in vivo. Eur. J. Appl. Physiol. Occup. Physiol. 71, 555-557. doi: 10.1007/BF00238560

Fung, Y. C. (1981). Biomechanics: Mechanical Properties of Living Tissues. New York, NY: Springer-Verlag.

Gatehouse, P. D., and Bydder, G. M. (2003). Magnetic resonance imaging of short T2 components in tissue. Clin. Radiol. 58, 1-19. doi: 10.1053/crad.2003.1157

Gautieri, A., Vesentini, S., Redaelli, A., and Buehler, M. J. (2012). Viscoelastic properties of model segments of collagen molecules. Matrix Biol. 31, 141-149. doi: 10.1016/j.matbio.2011.11.005

Gennisson, J. L., Deffieux, T., Fink, M., and Tanter, M. (2013). Ultrasound elastography: principles and techniques. Diagn. Interv. Imaging 94, 487-495. doi: 10.1016/j.diii.2013.01.022

Gennisson, J. L., Deffieux, T., Mace, E., Montaldo, G., Fink, M., and Tanter, M. (2010). Viscoelastic and anisotropic mechanical properties of in vivo muscle tissue assessed by supersonic shear imaging. Ultrasound Med. Biol. 36, 789-801. doi: 10.1016/j.ultrasmedbio.2010.02.013

Geremia, J. M., Bobbert, M. F., Casa Nova, M., Ott, R. D., Lemos Fde, A., Lupion Rde, O., et al. (2015). The structural and mechanical properties of the Achilles tendon 2 years after surgical repair. Clin. Biomech. 30, 485-492. doi: 10.1016/j.clinbiomech.2015.03.005

Glaser, K. J., Felmlee, J. P., and Ehman, R. L. (2006). Rapid MR elastography using selective excitations. Magn. Reson. Med. 55, 1381-1389. doi: 10.1002/mrm.20913

Guivel-Scharen, V., Sinnwell, T., Wolff, S. D., and Balaban, R. S. (1998). Detection of proton chemical exchange between metabolites and water in biological tissues. J. Magn. Reson. 133, 36-45. doi: 10.1006/jmre.1998.1440

Gupta, A., Li, W., Stebbins, G. T., Magin, R. L., and Wang, V. M. (2010). "High resolution diffusion tensor MRI of rabbit tendons and ligaments at 11.7 T," in Proceedings of the International Society for Magnetic Resonance in Medicine (ISMRM) (Stockholm), 885.

Han, M., Larson, P. E., Liu, J., and Krug, R. (2014). Depiction of achilles tendon microstructure in vivo using high-resolution 3-dimensional ultrashort echotime magnetic resonance imaging at 7 T. Invest. Radiol. 49, 339-345. doi: 10.1097/RLI.0000000000000025

He, X., Wengler, K., Sacher, A. C., Verastegui, M. A. O., Simeone, A., Huang, M., et al. (2016). "Diffusion tensor imaging of human Achilles tendon by stimulated echo RESOLVE (ste-RESOLVE)," in Proceedings of the International Society for Magnetic Resonance in Medicine (ISMRM) (Singapore), 367.

Helfenstein-Didier, C., Andrade, R. J., Brum, J., Hug, F., Tanter, M., Nordez, A., et al. (2016). In vivo quantification of the shear modulus of the human Achilles tendon during passive loading using shear wave dispersion analysis. Phys. Med. Biol. 61, 2485-2496. doi: 10.1088/0031-9155/61/6/2485

Helmer, K. G., Nair, G., Cannella, M., and Grigg, P. (2006). Water movement in tendon in response to a repeated static tensile load using onedimensional magnetic resonance imaging. J. Biomech. Eng. 128, 733-741. doi: $10.1115 / 1.2244573$

Hug, F., Lacourpaille, L., Maisetti, O., and Nordez, A. (2013). Slack length of gastrocnemius medialis and Achilles tendon occurs at different ankle angles. J. Biomech. 46, 2534-2538. doi: 10.1016/j.jbiomech.2013.07.015

Hug, F., Tucker, K., Gennisson, J. L., Tanter, M., and Nordez, A. (2015). Elastography for muscle biomechanics: toward the estimation of 
individual muscle force. Exerc. Sport Sci. Rev. 43, 125-133. doi: 10.1249/JES.0000000000000049

Juras, V., Apprich, S., Pressl, C., Zbyn, S., Szomolanyi, P., Domayer, S., et al. (2013a). Histological correlation of $7 \mathrm{~T}$ multi-parametric MRI performed in ex-vivo Achilles tendon. Eur. J. Radiol. 82, 740-744. doi: 10.1016/j.ejrad.2011.09.022

Juras, V., Apprich, S., Szomolanyi, P., Bieri, O., Deligianni, X., and Trattnig, S. (2013b). Bi-exponential T2 analysis of healthy and diseased Achilles tendons: an in vivo preliminary magnetic resonance study and correlation with clinical score. Eur. Radiol. 23, 2814-2822. doi: 10.1007/s00330-013-2897-8

Juras, V., Winhofer, Y., Szomolanyi, P., Vosshenrich, J., Hager, B., Wolf, P., et al. (2015). Multiparametric MR imaging depicts glycosaminoglycan change in the achilles tendon during ciprofloxacin administration in healthy men: initial observation. Radiology 275, 763-771. doi: 10.1148/radiol.15140484

Juras, V., Zbyn, S., Pressl, C., Domayer, S. E., Hofstaetter, J. G., Mayerhoefer, M. E., et al. (2012a). Sodium MR imaging of Achilles tendinopathy at $7 \mathrm{~T}$ : preliminary results. Radiology 262, 199-205. doi: 10.1148/radiol.11110897

Juras, V., Zbyn, S., Pressl, C., Valkovic, L., Szomolanyi, P., Frollo, I., et al. (2012b). Regional variations of $\mathrm{T}(2)^{*}$ in healthy and pathologic achilles tendon in vivo at 7 Tesla: preliminary results. Magn. Reson. Med. 68, 1607-1613. doi: $10.1002 / \mathrm{mrm} .24136$

Kjaer, M. (2004). Role of extracellular matrix in adaptation of tendon and skeletal muscle to mechanical loading. Physiol. Rev. 84, 649-698. doi: 10.1152/physrev.00031.2003

Kjaer, M., Langberg, H., Heinemeier, K., Bayer, M. L., Hansen, M., Holm, L., et al. (2009). From mechanical loading to collagen synthesis, structural changes and function in human tendon. Scand. J. Med. Sci. Sports 19, 500-510. doi: 10.1111/j.1600-0838.2009.00986.x

Klauser, A. S., Miyamoto, H., Bellmann-Weiler, R., Feuchtner, G. M., Wick, M. C., and Jaschke, W. R. (2014). Sonoelastography: musculoskeletal applications. Radiology 272, 622-633. doi: 10.1148/radiol.14121765

Kot, B. C., Zhang, Z. J., Lee, A. W., Leung, V. Y., and Fu, S. N. (2012). Elastic modulus of muscle and tendon with shear wave ultrasound elastography: variations with different technical settings. PLOS ONE 7:e44348. doi: 10.1371/journal.pone.0044348

Kubo, K., Akima, H., Kouzaki, M., Ito, M., Kawakami, Y., Kanehisa, H., et al. (2000). Changes in the elastic properties of tendon structures following 20 days bed-rest in humans. Eur. J. Appl. Physiol. 83, 463-468. doi: $10.1007 /$ s004210000309

Kubo, K., Akima, H., Ushiyama, J., Tabata, I., Fukuoka, H., Kanehisa, H., et al. (2004). Effects of 20 days of bed rest on the viscoelastic properties of tendon structures in lower limb muscles. Br. J. Sports Med. 38, 324-330. doi: 10.1136/bjsm.2003.005595

Kubo, K., Kanehisa, H., and Fukunaga, T. (2002a). Effect of stretching training on the viscoelastic properties of human tendon structures in vivo. J. Appl. Physiol. 92, 595-601. doi: 10.1152/japplphysiol.00658.2001

Kubo, K., Kanehisa, H., and Fukunaga, T. (2002b). Effects of resistance and stretching training programmes on the viscoelastic properties of human tendon structures in vivo. J. Physiol. (Lond). 538, 219-226. doi: 10.1113/jphysiol.2001.012703

Kubo, K., Kanehisa, H., and Fukunaga, T. (2003). Gender differences in the viscoelastic properties of tendon structures. Eur. J. Appl. Physiol. 88, 520-526. doi: 10.1007/s00421-002-0744-8

Kubo, K., Kawakami, Y., Kanehisa, H., and Fukunaga, T. (2002c). Measurement of viscoelastic properties of tendon structures in vivo. Scand. J. Med. Sci. Sports 12, 3-8. doi: 10.1034/j.1600-0838.2002.120102.x

Kubo, K., Teshima, T., Ikebukuro, T., Hirose, N., and Tsunoda, N. (2014). Tendon properties and muscle architecture for knee extensors and plantar flexors in boys and men. Clin. Biomech. 29, 506-511. doi: 10.1016/j.clinbiomech.2014.04.001

Lacourpaille, L., Nordez, A., Hug, F., Couturier, A., Dibie, C., and Guilhem, G. (2014). Time-course effect of exercise-induced muscle damage on localized muscle mechanical properties assessed using elastography. Acta Physiol. (Oxf). 211, 135-146. doi: 10.1111/apha.12272

Langberg, H., Rosendal, L., and Kjaer, M. (2001). Training-induced changes in peritendinous type I collagen turnover determined by microdialysis in humans. J. Physiol. (Lond). 534, 297-302. doi: 10.1111/j.1469-7793.2001. 00297.x
Le Sant, G., Ates, F., Brasseur, J. L., and Nordez, A. (2015). Elastography Study of Hamstring Behaviors during Passive Stretching. PLoS ONE 10:e139272. doi: 10.1371/journal.pone.0139272

Lenskjold, A., Kongsgaard, M., Larsen, J. O., Nielsen, R. H., Kovanen, V., Aagaard, P., et al. (2015). The influence of physical activity during youth on structural and functional properties of the Achilles tendon. Scand. J. Med. Sci. Sports 25, 25-31. doi: 10.1111/sms.12143

Lichtwark, G. A., Cresswell, A. G., Ker, R. F., Reeves, N. D., Maganaris, C. N., Magnusson, S. P., et al. (2013). Commentaries on viewpoint: on the hysteresis in the human Achilles tendon. J. Appl. Physiol. 114, 518-520. doi: 10.1152/japplphysiol.01525.2012

Ma, Y., Carl, M., Bydder, G. M., and Du, J. (2016). "3D ultrshort echo time cones sequence with diffusion weighted imaging (3D UTE-Cones-DWI): evaluation of the angular dependence of diffusion in the Achilles tendon," in Proceedings of the International Society for Magnetic Resonance in Medicine (ISMRM) (Singapore), 3025.

Mademli, L., and Arampatzis, A. (2008). Mechanical and morphological properties of the triceps surae muscle-tendon unit in old and young adults and their interaction with a submaximal fatiguing contraction. J. Electromyogr. Kinesiol. 18, 89-98. doi: 10.1016/j.jelekin.2006.09.008

Maganaris, C. N. (2002). Tensile properties of in vivo human tendinous tissue. J. Biomech. 35, 1019-1027. doi: 10.1016/S0021-9290(02)00047-7

Maganaris, C. N. (2005). Validity of procedures involved in ultrasound-based measurement of human plantarflexor tendon elongation on contraction. $J$. Biomech. 38, 9-13. doi: 10.1016/j.jbiomech.2004.03.024

Maganaris, C. N., and Paul, J. P. (2000). Hysteresis measurements in intact human tendon. J. Biomech. 33, 1723-1727. doi: 10.1016/S0021-9290(00)00130-5

Maganaris, C. N., and Paul, J. P. (2002). Tensile properties of the in vivo human gastrocnemius tendon. J. Biomech. 35, 1639-1646. doi: 10.1016/S00219290(02)00240-3

Magnusson, P. (2002). Ultrasonography, exploration of human muscle-tendon function. Scand. J. Med. Sci. Sports 12, 1-2. doi: 10.1034/k.1600-0838.2002.120102.x

Magnusson, S. P., Beyer, N., Abrahamsen, H., Aagaard, P., Neergaard, K., and Kjaer, M. (2003a). Increased cross-sectional area and reduced tensile stress of the Achilles tendon in elderly compared with young women. J. Gerontol. Series A Biol. Sci. Med. Sci. 58, 123-127. doi: 10.1093/gerona/58.2.B123

Magnusson, S. P., Hansen, M., Langberg, H., Miller, B., Haraldsson, B., Westh, E. K., et al. (2007). The adaptability of tendon to loading differs in men and women. Int. J. Exp. Pathol. 88, 237-240. doi: 10.1111/j.1365-2613.2007.00551.x

Magnusson, S. P., Hansen, P., Aagaard, P., Brond, J., Dyhre-Poulsen, P., Bojsen-Moller, J., et al. (2003b). Differential strain patterns of the human gastrocnemius aponeurosis and free tendon, in vivo. Acta Physiol. Scand. 177, 185-195. doi: 10.1046/j.1365-201X.2003.01048.x

Mariappan, Y. K., Glaser, K. J., and Ehman, R. L. (2010). Magnetic resonance elastography: a review. Clin. Anat. 23, 497-511. doi: 10.1002/ca.21006

Momot, K. I., Pope, J. M., and Wellard, R. M. (2010). Anisotropy of spin relaxation of water protons in cartilage and tendon. NMR Biomed. 23, 313-324. doi: $10.1002 / \mathrm{nbm} .1466$

Morse, C. I., Degens, H., Seynnes, O. R., Maganaris, C. N., and Jones, D. A. (2008). The acute effect of stretching on the passive stiffness of the human gastrocnemius muscle tendon unit. J. Physiol. (Lond). 586, 97-106. doi: 10.1113/jphysiol.2007.140434

Muthupillai, R., Lomas, D. J., Rossman, P. J., Greenleaf, J. F., Manduca, A., and Ehman, R. L. (1995). Magnetic resonance elastography by direct visualization of propagating acoustic strain waves. Science 269, 1854-1857. doi: $10.1126 /$ science.7569924

Nordez, A., Fouré, A., Dombroski, E. W., Mariot, J. P., Cornu, C., and Mcnair, P. J. (2010). Improvements to Hoang et al.'s method for measuring passive length-tension properties of human gastrocnemius muscle in vivo. J. Biomech. 43, 379-382. doi: 10.1016/j.jbiomech.2009.07.034

Papazoglou, S., Braun, J., Hamhaber, U., and Sack, I. (2005). Two-dimensional waveform analysis in MR elastography of skeletal muscles. Phys. Med. Biol. 50, 1313-1325. doi: 10.1088/0031-9155/50/6/018

Parkinson, J., Samiric, T., Ilic, M. Z., Cook, J., Feller, J. A., and Handley, C. J. (2010). Change in proteoglycan metabolism is a characteristic of human patellar tendinopathy. Arthritis Rheum. 62, 3028-3035. doi: 10.1002/art. 27587 
Pingel, J., Lu, Y., Starborg, T., Fredberg, U., Langberg, H., Nedergaard, A., et al. (2014). 3-D ultrastructure and collagen composition of healthy and overloaded human tendon: evidence of tenocyte and matrix buckling. J. Anat. 224, 548-555. doi: 10.1111/joa.12164

Provost, J., Papadacci, C., Arango, J. E., Imbault, M., Fink, M., Gennisson, J. L., et al. (2014). 3D ultrafast ultrasound imaging in vivo. Phys. Med. Biol. 59, L1-L13. doi: 10.1088/0031-9155/59/19/L1

Regatte, R. R., Akella, S. V., Borthakur, A., Kneeland, J. B., and Reddy, R. (2003). In vivo proton MR three-dimensional T1rho mapping of human articular cartilage: initial experience. Radiology 229, 269-274. doi: 10.1148/radiol.2291021041

Roberts, T. J., Marsh, R. L., Weyand, P. G., and Taylor, C. R. (1997). Muscular force in running turkeys: the economy of minimizing work. Science 275, 1113-1115. doi: $10.1126 /$ science. 275.5303 .1113

Robson, M. D., Benjamin, M., Gishen, P., and Bydder, G. M. (2004). Magnetic resonance imaging of the Achilles tendon using ultrashort TE (UTE) pulse sequences. Clin. Radiol. 59, 727-735. doi: 10.1016/j.crad.2003.11.021

Samiric, T., Parkinson, J., Ilic, M. Z., Cook, J., Feller, J. A., and Handley, C. J. (2009). Changes in the composition of the extracellular matrix in patellar tendinopathy. Matrix Biol. 28, 230-236. doi: 10.1016/j.matbio.2009.04.001

Sarman, H., Atmaca, H., Cakir, O., Muezzinoglu, U. S., Anik, Y., Memisoglu, $\mathrm{K}$., et al. (2015). Assessment of postoperative tendon quality in patients with achilles tendon rupture using diffusion tensor imaging and tendon fiber tracking. J. Foot Ankle Surg. 54, 782-786. doi: 10.1053/j.jfas.2014.12.025

Schmitt, B., Zbyn, S., Stelzeneder, D., Jellus, V., Paul, D., Lauer, L., et al. (2011). Cartilage quality assessment by using glycosaminoglycan chemical exchange saturation transfer and (23) Na MR imaging at 7 T. Radiology 260, 257-264. doi: 10.1148/radiol.11101841

Schreiner, M. M., Zbyn, S., Schmitt, B., Weber, M., Domayer, S., Windhager, R., et al. (2016). Reproducibility and regional variations of an improved gagCEST protocol for the in vivo evaluation of knee cartilage at 7 T. MAGMA 29, 513-521. doi: 10.1007/s10334-016-0544-5

Seynnes, O. R., Bojsen-Moller, J., Albracht, K., Arndt, A., Cronin, N. J., Finni, T., et al. (2015). Ultrasound-based testing of tendon mechanical properties: a critical evaluation. J. Appl. Physiol. 118, 133-141. doi: 10.1152/japplphysiol.00849.2014

Silver, F. H., Ebrahimi, A., and Snowhill, P. B. (2002). Viscoelastic properties of self-assembled type I collagen fibers: molecular basis of elastic and viscous behaviors. Connect. Tissue Res. 43, 569-580. doi: 10.1080/03008200290001302

Slane, L. C., DeWall, R., Martin, J., Lee, K., and Thelen, D. G. (2015). Middle-aged adults exhibit altered spatial variations in Achilles tendon wave speed. Physiol. Meas. 36, 1485-1496. doi: 10.1088/0967-3334/36/7/1485

Slane, L. C., Martin, J., DeWall, R., Thelen, D., and Lee, K. (2016). Quantitative ultrasound mapping of regional variations in shear wave speeds of the aging Achilles tendon. Eur. Radiol. doi: 10.1007/s00330-016-4409-0. [Epub ahead of print].

Song, H. K., and Wehrli, F. W. (1998). Variable TE gradient and spin echo sequences for in vivo MR microscopy of short T2 species. Magn. Reson. Med. 39, 251-258. doi: 10.1002/mrm.1910390212
Stephenson, A. L., Wu, W., Cortes, D., and Rochon, P. A. (2013). Tendon injury and fluoroquinolone use: a systematic review. Drug Saf. 36, 709-721. doi: 10.1007/s40264-013-0089-8

Thompson, J. I., and Czernuszka, J. T. (1995). The effect of two types of crosslinking on some mechanical properties of collagen. Biomed. Mater. Eng. 5, $37-48$.

Thorpe, C. T., Udeze, C. P., Birch, H. L., Clegg, P. D., and Screen, H. R. (2012). Specialization of tendon mechanical properties results from interfascicular differences. J. R. Soc. Interface 9, 3108-3117. doi: 10.1098/rsif.20 12.0362

Trattnig, S., Bogner, W., Gruber, S., Szomolanyi, P., Juras, V., Robinson, S., et al. (2015). Clinical applications at ultrahigh field $(7 \mathrm{~T})$. Where does it make the difference? NMR Biomed. doi: 10.1002/nbm.3272. [Epub ahead of print].

Trattnig, S., Zbyn, S., Schmitt, B., Friedrich, K., Juras, V., Szomolanyi, P., et al. (2012). Advanced MR methods at ultra-high field (7 Tesla) for clinical musculoskeletal applications. Eur. Radiol. 22, 2338-2346. doi: 10.1007/s00330012-2508-0

Turan, A., Teber, M. A., Yakut, Z. I., Unlu, H. A., and Hekimoglu, B. (2015), Sonoelastographic assessment of the age-related changes of the Achilles tendon. Med. Ultrason. 17, 58-61. doi: 10.11152/mu.2013.2066.171.ayt

van der Rijt, J. A., van der Werf, K. O., Bennink, M. L., Dijkstra, P. J., and Feijen, J. (2006). Micromechanical testing of individual collagen fibrils. Macromol. Biosci. 6, 697-702. doi: 10.1002/mabi.200600063

Wang, J. H. (2006). Mechanobiology of tendon. J. Biomech. 39, 1563-1582. doi: 10.1016/j.jbiomech.2005.05.011

Wang, L., and Regatte, R. R. (2015). T(1)rho MRI of human musculoskeletal system. J. Magn. Reson. Imaging 41, 586-600. doi: 10.1002/jmri. 24677

Wellen, J., Helmer, K. G., Grigg, P., and Sotak, C. H. (2005). Spatial characterization of $\mathrm{T} 1$ and $\mathrm{T} 2$ relaxation times and the water apparent diffusion coefficient in rabbit Achilles tendon subjected to tensile loading. Magn. Reson. Med. 53, 535-544. doi: 10.1002/mrm.20361

Westh, E., Kongsgaard, M., Bojsen-Moller, J., Aagaard, P., Hansen, M., Kjaer M., et al. (2008). Effect of habitual exercise on the structural and mechanical properties of human tendon, in vivo, in men and women. Scand. J. Med. Sci. Sports 18, 23-30. doi: 10.1111/j.1600-0838.2007.00638.x

Yoon, J. H., and Halper, J. (2005). Tendon proteoglycans: biochemistry and function. J. Musculoskelet. Neuronal Interact. 5, 22-34.

Conflict of Interest Statement: The author declares that the research was conducted in the absence of any commercial or financial relationships that could be construed as a potential conflict of interest.

Copyright $\odot 2016$ Fouré. This is an open-access article distributed under the terms of the Creative Commons Attribution License (CC BY). The use, distribution or reproduction in other forums is permitted, provided the original author(s) or licensor are credited and that the original publication in this journal is cited, in accordance with accepted academic practice. No use, distribution or reproduction is permitted which does not comply with these terms. 